\title{
CDISC CDASH Exposure Dosing Frequency per Interval Terminology
}

National Cancer Institute

\section{Source}

National Cancer Institute. CDISC CDASH Exposure Dosing Frequency per Interval

Terminology. NCl Thesaurus. Code C78745.

A terminology subset of the CDISC Study Data Tabulation Model (SDT M) Frequency

codelist created for Clinical Data Acquisition Standards Harmonization (CDASH) Exposure Dosing Frequency per Interval terminology. 